\title{
CHronic Rhinosinusitis Outcome MEasures (CHROME) - developing a core outcome set for trials of interventions in chronic rhinosinusitis*
}

\author{
Claire Hopkins' ${ }^{1}$, Roland Hettige ${ }^{2}$, Archana Soni-Jaiswal ${ }^{3}$, Raj Lakhani ${ }^{4}$, Sean \\ Carrie $^{5}$, Anders Cervin ${ }^{6}$, Richard Douglas ${ }^{7}$, Wytske Fokkens ${ }^{8}$, Richard Harvey ${ }^{9}$, \\ Peter Hellings ${ }^{10}$, Andreas Leunig ${ }^{11}$, Valerie Lund ${ }^{12}$, Carl Philpott ${ }^{13}$, Tim Smith ${ }^{14}$, \\ De-Yun Wang ${ }^{15}$, Luke Rudmik' ${ }^{16}$
}

Rhinology 56: 22-32, 2018

https://doi.org/10.4193/Rhino17.247

*Received for publication:

December 10, 2017

${ }^{1}$ ENT Department, Guy's Hospital, London, United Kingdom; ${ }^{2}$ Wexham Park Hospital, Wexham, United Kingdom; ${ }^{3}$ Manchester Royal Accepted: December 17,2017 Infirmary, Manchester, London, United Kingdom; ${ }^{4}$ St George's Hospital, London, United Kingdom; ${ }^{5}$ Freeman Hopsital, Newcastle, United Kingdom; ${ }^{6}$ Royal Brisbane Hospital, Brisbane, Australia; ${ }^{7}$ Auckland City Hospital, Auckland, New Zealand; ${ }^{8}$ Academic Medical Centre, Amsterdam, The Netherlands; ${ }^{9}$ St Vincent's Hospital, Sydney, Australia; ${ }^{10}$ Univerity Hospital Leuven, Leuven Belgium; ${ }^{11}$ HNO-Klinik München-Bogenhausen, Munich, Germany; ${ }^{12}$ Royal National Throat Nose and Ear Hospital, London, United Kingdom; ${ }^{13}$ University of Easy Anglia, Norwich, United Kingdom; ${ }^{14}$ Oregon Sinus Centre, Portland, OR, USA; ${ }^{15}$ Yong Loo Lin School of Medicine, Singapore, Singapore; ${ }^{16}$ University of Calgary, Calgary, Canada

\begin{abstract}
Statement of problem: Evaluating the effectiveness of treatments in chronic rhinosinusitis (CRS) have been limited by both a paucity of high quality randomised trials, and the heterogeneity of outcomes in those that have been reported.

Core outcome sets (COS) are an agreed, standardized set of outcomes that should be measured and reported by future trials as a minimum and will facilitate future meta-analysis of trial results in systematic reviews (SRs). We set out to develop a core outcome set for interventions for adults with CRS.
\end{abstract}

Method(s) of study: A long-list of potential outcomes was identified by a steering group utilising a literature review, thematic analysis of a wide range of stakeholders' views and systematic analysis of currently available Patient Reported Outcome Measures (PROMs). A subsequent e-Delphi process allowed 110 patients and healthcare practitioners to individually rate the outcomes in terms of importance, on a Likert scale.

Main results: After 2 rounds of the iterative Delphi process, the 54 initial outcomes were distilled down to a final core-outcome set of 15 items, over 4 domains.

Principal conclusions: The authors hope inclusion of these core outcomes in future trials will increase the value of research on interventions for CRS in adults. It was felt important to make recommendations regarding how these outcomes should be measured, although additional work is now required to further develop and revalidate existing outcome measures.

Key words: chronic rhinosinusitis, outcome measurement, effectiveness trials, core outcome set

\section{Aim \\ To develop a core outcome set of outcome measures for trials evaluating the effectiveness of treatment interventions for adults with chronic rhinosinusitis.}

\section{Introduction}

CRS represents a common and widespread source of ill health, with $11 \%$ of UK adults reporting CRS symptoms in a worldwide population study ${ }^{(1)}$. CRS has been shown to have a major impact on quality of life that is reportedly greater in several domains of the SF-36 than angina or chronic respiratory disease (2). Acute exacerbations, inadequate symptom control and respiratory disease exacerbation are common amongst this population. Longitudinal primary care data from the Clinical Practice Re- 
search Datalink (CPRD) data shows that $1 \%$ of UK adults receive treatment from their GP each year with an average of 4 GP visits per year, receiving multiple prescribed medical treatments and with $91 \%$ of rhinosinusitis patients receiving an antibiotic prescription ${ }^{(3)}$. There is a significant cost of the disease to the patient and healthcare systems, but also significant indirect costs through absenteeism and presenteeism. The total direct cost attributed to management of CRS is estimated to reach $\$ 11$ billion per year in the USA ${ }^{(4)}$. An earlier systematic review showed that the direct healthcare costs in the USA attributed to CRS is approximately $\$ 6.9-9.9$ billion per annum, with indirect cost to society through lost productivity estimated at $\$ 13$ billion per annum ${ }^{(5)}$

There is considerable variation in current practice, both in terms of antibiotic prescribing and surgical intervention rates $(6,7)$. This relates, in part, to the lack of strong recommendations in treatment guidelines. Recently, a suite of Cochrane reviews evaluating the effectiveness of treatments in CRS have been published, but they are limited both by a paucity of high quality randomised trials, and the heterogeneity of outcomes in those that have been reported, which precludes meta-analysis.

Core outcome sets (COS) are an agreed, standardized set of outcomes that should be measured and reported by future trials as a minimum, and are not meant to be restrictive if trialists wish to include additional outcomes. However, inclusion of the core outcomes will facilitate future meta-analysis of trial results in systematic reviews (SRs). The heterogeneity of outcomes currently reported restricts our ability to combine results, and use of a COS may maximize the potential for a trial to contribute to future SRs. Furthermore, the use of core outcome sets also aims to reduce outcome reporting bias, where outcomes are measured but left unreported, often in the setting of negative findings.

The use of a COS is supported by the World Health Organisation (8) and the Cochrane Group for use in reviews of the effects of healthcare interventions, and they have already been developed and adopted by multiple medical and surgical specialties ${ }^{(9)}$. The development of core outcome sets has been promoted and supported by the COMET (Core Outcome Measures in Effectiveness Trials) Initiative. The success of a COS is likely to be related to its simplicity, and an ideal of 3-4 main outcome measures were anticipated to be included in the final COS for CRS. Of course, due to the heterogeneity of symptoms that patients with CRS experience and the impact on their quality of life, restricting it to only a few outcomes was likely to be challenging. It should consider both benefits and harms, and initially the core outcome set should consider 'what' to measure, rather than 'how' to measure it. Although considering trials of effectiveness, the outcomes selected are likely to be relevant to routine clinical practice. In

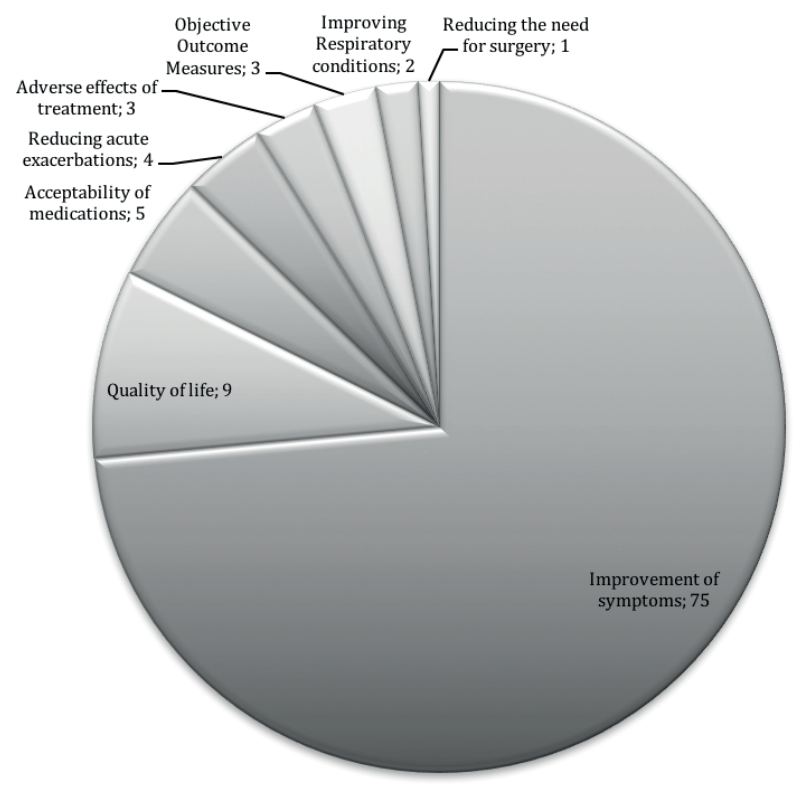

Figure 1. Responses to OMIPP pilot project, grouped under main domains, $\%$.

rheumatology, the OMERACT group has developed 9 sets over the last 9 years, and now $70 \%$ of clinical trials report the COS.

Although there is no specific methodology, the majority of core outcome sets follow a process of identifying existing knowledge to develop a long list of outcomes, followed by consensus methods using a Delphi process to achieve global agreement between different stakeholder groups, as recommended by COMET ${ }^{(10)}$.

\section{Materials and methods}

cos development registration

The project was registered with the Core Outcome Measures in Effectiveness Trials (COMET) Register, and the development process followed guidance issued by COMET. In particular the 11 minimum standards for COS development ${ }^{(11)}$ were met and the checklist for reporting COS study reporting was followed ${ }^{(12)}$.

Ethical approval was granted for patient participation in the focus group and Delphi process. Funding was awarded by the British Rhinologic Society to support the COS development.

\section{Defining scope}

A steering group of ENT surgeons active in clinical research in the field of CRS was assembled to consider the scope and design of the COS. It was agreed to consider all interventions for CRS, in adult patients (over 18 years). The COS is primarily aimed at research but it was felt that it would also be suitable for routine clinical care. 
Table 1. Patient demographics, pre-operative clinical and laboratory tests.

\begin{tabular}{|c|c|c|}
\hline Category & Core-category & Specific outcome/outcome measure used by trials \\
\hline $\begin{array}{l}\text { Changes in patient } \\
\text { rated symptom } \\
\text { severity }\end{array}$ & $\begin{array}{l}\text { Global } \\
\text { Disease specific }\end{array}$ & $\begin{array}{l}\text { VAS (multiple different non-validated scales used), patient diary } \\
\text { VAS for nasal blockage/congestion, nasal discharge, olfactory disturbance, facial pain/pres- } \\
\text { sure, headache, itching, sneezing, ocular symptoms. Clinical history for snoring, rhinitis, nasal } \\
\text { obstruction, headache, dry mouth, loss of smell, use of medication }\end{array}$ \\
\hline Quality of life & $\begin{array}{l}\text { Global } \\
\text { Disease specific }\end{array}$ & $\begin{array}{l}\text { SF-36, SF-12, } 7 \text { point scale } \\
\text { SNOT 20/21/22, RSDI, RSOM-31, SNAQ-11, Chronic sinusitis survey }\end{array}$ \\
\hline \multirow{6}{*}{$\begin{array}{l}\text { Physiological as- } \\
\text { sessments }\end{array}$} & Endoscopic & $\begin{array}{l}\text { VAS for adhesions, stenosis of ostia, patency of frontal recess, blood crusts, turbinate size. } \\
\text { Polyp size using the Malm, Lindholt or Lund-Mackay scores. Descriptive text about mucosal } \\
\text { oedema, discharge, crusting, scarring and swelling }\end{array}$ \\
\hline & Radiological & CT using Lund-Mackay scores or Catalano and Payne for the frontal recess, MRI, X-ray \\
\hline & Nasal Airflow & PNIF, PNEF \\
\hline & Mucociliary Function & Saccharin clearance testing \\
\hline & Olfactory testing & $\begin{array}{l}\text { VAS scores, butanol threshold testing, carbinol sniff bottles, UPSIT, individual odours such as } \\
\text { coffee, turpentine and lavender }\end{array}$ \\
\hline & Sinus manometry & Active anterior rhinomanometry, acoustic anterior and posterior rhinomanometry \\
\hline \multirow{2}{*}{ Microbiological } & Microbiome & $\begin{array}{l}\text { Antral fluid culture, lavage culture, nasal swabs, Alternaria protein levels, middle meatus swab, } \\
\text { nasal discharge swab }\end{array}$ \\
\hline & Fungal Hyphae & As above \\
\hline \multirow{3}{*}{$\begin{array}{l}\text { Lower Airway } \\
\text { Disease }\end{array}$} & $\begin{array}{l}\text { Patient reported outcome } \\
\text { measures }\end{array}$ & Not assessed by any trial \\
\hline & Patient symptoms & VAS for SOB, cough, wheeze, breathlessness, need for a B2 agonist \\
\hline & Respiratory function tests & FEV1, FVC, VC, MEF50, histamine inhalation challenge, PEFR, exhaled NO \\
\hline \multirow{2}{*}{ Side Effects } & Medical & $\begin{array}{l}\text { Patient diary, epistaxis rates, plasma cortisol levels, serum ACTH, Ophthalmological ocular as- } \\
\text { sessment, urinary cortisol levels, oral candidiasis }\end{array}$ \\
\hline & Surgical & Epistaxis, synechia, sinonasal infections, revision surgery \\
\hline \multirow{5}{*}{$\begin{array}{l}\text { Acceptability of } \\
\text { treatment }\end{array}$} & Compliance & VAS, patient diary, empty medicine containers \\
\hline & Acceptability to patients & Discomfort, length of improvement \\
\hline & Cost incurred by patient & Not assessed by any trial \\
\hline & Cost to third party & Not assessed by any trial \\
\hline & Patient preference & Based on history from patient in only one RCT \\
\hline Biomarkers & & $\begin{array}{l}\text { Nitric oxide, IL-8, IL-5, IL-4, serum eosinophils, mucous eosinophil derived neurostosin, } a-2 \\
\text { macroglobulin, IL- } \beta \text {, TNF- } a \text { in nasal lavage, mucosal biopsy for CD4, IL-4, MBP and T-cells. Skin } \\
\text { allergy testing, serum IgE, ESR, WCC, CRP }\end{array}$ \\
\hline Other & & Need for rescue medication, rates of revision surgery \\
\hline
\end{tabular}

\section{Stakeholder involvement}

Both patients, researchers in CRS and physicians (including ENT specialists, Allergists, Respiratory physicians and primary care physicians) were involved in every stage of COS development, including defining scope, developing the long-list, the eDelphi process and review and analysis of the final results.

A Steering Group was convened, consisting of authors of the European Position Paper on Rhinosinusitis and Nasal Polyps (EPOS) Guideline, all of whom are actively engaged in research in the field of CRS and are practicing ENT surgeons. A patient focus group was formed with patients recruited from clinical practice under the care of the lead author. Patients were selected with and without nasal polyps and at different stages of their patient journey.

\section{Long-list development}

A long-list of potential core outcomes was drawn up from a number of sources; 
Table 2. Final long-list of items included in the Delphi process.

\begin{tabular}{|c|c|}
\hline Patient reported symptoms & $\begin{array}{l}\text { Overall symptom severity } \\
\text { Frequency of symptoms } \\
\text { Complete resolution of symptoms } \\
\text { Duration of symptoms } \\
\text { Duration of treatment effect }\end{array}$ \\
\hline Individual symptoms & $\begin{array}{l}\text { Sense of smell } \\
\text { Runny nose / Nasal discharge (anterior or posterior) } \\
\text { Nasal obstruction / blockage/congestion } \\
\text { Facial pain / pressure } \\
\text { Headache } \\
\text { Sleep disturbance } \\
\text { Fatigue } \\
\text { Itching / Sneezing } \\
\text { Epistaxis } \\
\text { Eye symptoms - dryness, itching, irritation } \\
\text { Ear symptoms - dizziness, fullness } \\
\text { Cough } \\
\text { Bad taste or bad breath } \\
\text { Dryness (nose or mouth) }\end{array}$ \\
\hline Control of disease & $\begin{array}{l}\text { Overall control of disease } \\
\text { Need for surgery } \\
\text { Occurrence of acute exacerbations } \\
\text { Ongoing treatment / healthcare utilisation }\end{array}$ \\
\hline Quality of Life & $\begin{array}{l}\text { Global quality of life } \\
\text { Disease specific quality of life } \\
\text { Impact on emotional wellbeing } \\
\text { Mental ill-health } \\
\text { Ability to perform normal activities }\end{array}$ \\
\hline Acceptability of treatment and side effects & $\begin{array}{l}\text { Compliance with treatment } \\
\text { Acceptability of treatment } \\
\text { Costs of treatment to patients } \\
\text { Costs of treatment to third party payers } \\
\text { Side effects of treatment (including medical and surgical) } \\
\text { Treatment preference }\end{array}$ \\
\hline $\begin{array}{l}\text { Impact on lower respiratory (chest) conditi- } \\
\text { ons (including Asthma) }\end{array}$ & $\begin{array}{l}\text { Lower respiratory (chest) symptoms } \\
\text { Clinical measures of lower respiratory (chest) function } \\
\text { Medication usage for lower respiratory (chest) disease } \\
\text { Hospitalisation for chest disease }\end{array}$ \\
\hline Clinical measures of the nose and sinuses & $\begin{array}{l}\text { Endoscopic appearances (including presence/quality of pus, presence and size of polyps, oedema, } \\
\text { crusting, inflammation) } \\
\text { Radiological evaluation (by repeated CT / MRI imaging) } \\
\text { Evaluation of nasal airway using special tests (using either acoustic rhinometry/rhinomanometry/ } \\
\text { PNIF/rhinospirometry) } \\
\text { Evaluation of mucociliary function (including saccharine testing/microscopy } \\
\text { Evaluation of sense of smell (olfactometry / Sniffin Sticks / UPSIT / Zurich/ etc.) } \\
\text { Maxillary sinus manometry }\end{array}$ \\
\hline Microbiological & $\begin{array}{l}\text { Changes in the individual Microbiome } \\
\text { Resistance to antibiotics in the community } \\
\text { Presence of fungal hyphae }\end{array}$ \\
\hline Biomarkers & $\begin{array}{l}\text { Eosinophil levels in serum } \\
\text { IgE levels in serum } \\
\text { Evaluation of exhaled nitric oxide in upper and lower respiratory tract } \\
\text { Mucosal Biopsies / histopathological specimens } \\
\text { Evaluation of nasal lavage } \\
\text { Serum Markers (IL-5, IL-8, TNF } \alpha \text {, IL-4, ESR.....) - can be defined at a later stage }\end{array}$ \\
\hline
\end{tabular}

\section{1) Systematic search of outcomes used in Cochrane Systematic} Reviews

A systematic search of outcomes used in randomised controlled trials included in Cochrane Systematic Reviews of interventions for CRS in adults or the Cochrane Central Register of Controlled Trials, was performed. All trials and reviews in adult patients were considered, including those in unselected CRS, those with and without polyps, and those with subtypes such as aspirin exacerbated respiratory disease and allergic fungal rhinosinusitis.

The full text articles for the Cochrane systematic reviews were obtained and analysed for eligibility by two independent revie- 
Table 3. Round 1 responses.

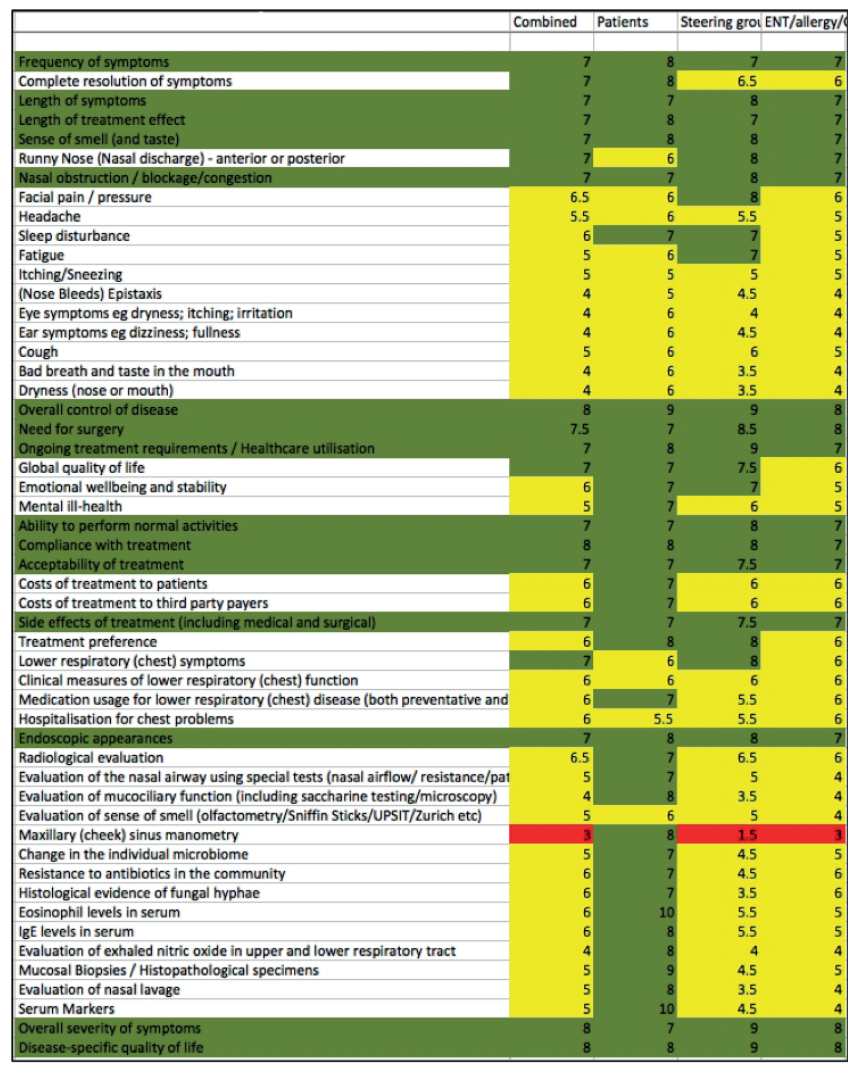

Median score for cohort and by stakeholder group, column $1=$ whole cohort, 2=patients, $3=$ steering group, $4=$ all other HCPs. Items deemed important by all stakeholder groups after round 1 of Delphi highlighted in green.
Table 4. Stability of median responses between rounds 1 and 2 .

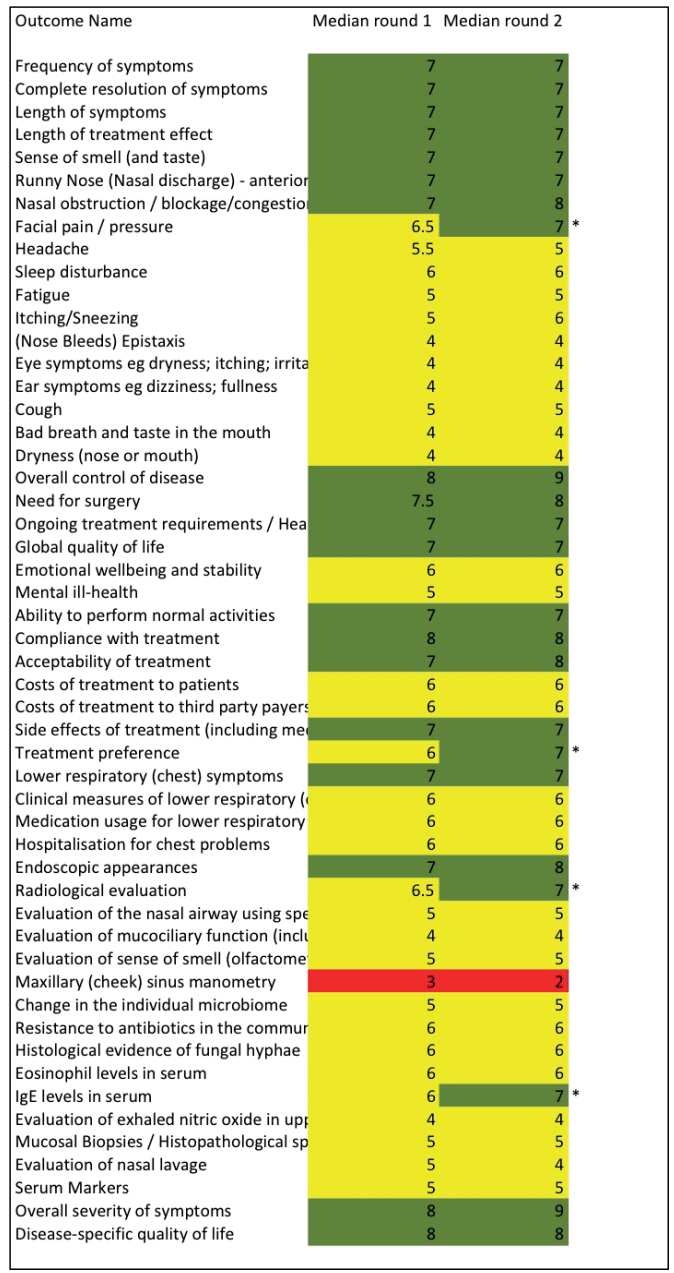

wers (ASJ and RL). As each systematic review presents the results of a number of pooled clinical trials, the published papers for each individual trial were obtained. The bibliographies of the trial papers were further evaluated to identify additional studies for inclusion, not identified within the Cochrane systematic reviews themselves.

Two independent reviewers extracted data from all identified randomised trials. If there was uncertainty or disagreement amongst them, a third reviewer was consulted and had the final say. Each individual outcome was mapped to a core subcategory. These sub-categories were then grouped into core categories. This process has been previously published, with the full methodology and results ${ }^{(13)}$.

\section{2) Outcomes important to Patients, Practitioners and Provi- ders (OMIPPP)}

This pilot project was commissioned by Cochrane UK to identify important outcomes interventions for CRS in adults ${ }^{(14)}$. It sought the views of a wide range of practitioners $(n=155)$ identified by professional societies and patients $(n=80)$, identified using a social media campaign. Using direct emailing, social media and printed cards, an online survey was distributed to a wide range of people involved in the care of patients with CRS. Patients and practitioners were asked to list the 3 outcomes from treatments most important to them. Responses were analysed through development of a thematic framework based on the data.

\section{3) Systematic review of currently available Patient Rated Out- come Measures (PROMs)}

A systematic review previously undertaken in 2015 assessed all published PROMS for CRS ${ }^{(15)}$. All included instruments were screened by 2 independent reviewers (ASJ and RL) to search for items or domains not included in the long list by the first two steps.

\section{4) Patient and Steering Group review}

Potential missing items were considered by a patient focus group, and the COS Steering Group, with particular reference to outcomes included in the EPOS guidelines ${ }^{(16)}$. The long-list was then compiled and mapped to simplified domains for use in the 
Table 5. Round 2 median scores by stakeholder group.

\begin{tabular}{|c|c|c|c|c|}
\hline \multirow[b]{2}{*}{ Frequency of symptoms } & Combined & Patients & \multicolumn{2}{|c|}{ Steering groı ENT/allergy/ } \\
\hline & 7 & 6.5 & 7.5 & 7 \\
\hline Complete resolution of symptoms & 7 & 7 & 7 & 7 \\
\hline Length of symptoms & 7 & 8 & 8 & 7 \\
\hline Length of treatment effect & 7 & 8 & 8 & 7 \\
\hline Sense of smell (and taste) & 7 & 9 & 8 & 7 \\
\hline Runny Nose (Nasal discharge) - anterior or posterior & 7 & 7.5 & 8 & 7 \\
\hline Nasal obstruction / blockage/congestion & 8 & 8 & 8 & \\
\hline Facial pain / pressure & 7 & 6.5 & 7.5 & \\
\hline Headache & 5 & 6 & 5 & \\
\hline Sleep disturbance & 6 & 6.5 & 7 & 6 \\
\hline Fatigue & 5 & 6.5 & 7 & 5 \\
\hline Itching/Sneezing & 6 & 6.5 & 5.5 & 5 \\
\hline (Nose Bleeds) Epistaxis & 4 & 5.5 & 3.5 & 4 \\
\hline Eye symptoms eg dryness; itching; irritation & 4 & 7 & 4 & 4 \\
\hline Ear symptoms eg dizziness; fullness & 4 & 6 & 4.5 & 4 \\
\hline Cough & 5 & 6.5 & 6.5 & 4 \\
\hline Bad breath and taste in the mouth & 4 & 5.5 & 4 & 4 \\
\hline Dryness (nose or mouth) & 4 & 6.5 & 4.5 & \\
\hline Overall control of disease & 9 & 8 & 9 & \\
\hline Need for surgery & 8 & 7 & 8.5 & \\
\hline Ongoing treatment requirements / Healthcare utilisation & 7 & 8 & 9 & 7 \\
\hline Global quality of life & 7 & 7 & 7.5 & \\
\hline Emotional wellbeing and stability & 6 & 7 & 7 & \\
\hline Mental ill-health & 5 & 6.5 & 6.5 & \\
\hline Ability to perform normal activities & 7 & 7 & 8 & \\
\hline Compliance with treatment & 8 & 8.5 & 8 & \\
\hline Acceptability of treatment & 8 & 8 & 8 & \\
\hline Costs of treatment to patients & 6 & 6 & 7 & \\
\hline Costs of treatment to third party payers & 6 & 6 & 7 & 6 \\
\hline Side effects of treatment (including medical and surgical) & 7 & 8 & 8 & \\
\hline Treatment preference & 7 & 7 & 7.5 & 7 \\
\hline Lower respiratory (chest) symptoms & 7 & 7.5 & 8 & \\
\hline Clinical measures of lower respiratory (chest) function & 6 & 7 & 6.5 & \\
\hline Medication usage for lower respiratory (chest) disease (both & 6 & 6.5 & 7 & \\
\hline Hospitalisation for chest problems & 6 & 7.5 & 7 & 6 \\
\hline Endoscopic appearances & 8 & 8.5 & 8.5 & 8 \\
\hline Radiological evaluation & 7 & 8.5 & 7 & \\
\hline Evaluation of the nasal airway using special tests (nasal airflc & 5 & 6 & 5 & \\
\hline Evaluation of mucociliary function (including saccharine test & 4 & 9 & 4 & \\
\hline Evaluation of sense of smell (olfactometry/Sniffin Sticks/UPS & 5 & 6.5 & 6.5 & \\
\hline Maxillary (cheek) sinus manometry & 2 & 4.5 & 1 & \\
\hline Change in the individual microbiome & 5 & 5.5 & 5 & 5 \\
\hline Resistance to antibiotics in the community & 6 & 6.5 & 6 & 6 \\
\hline Histological evidence of fungal hyphae & 6 & 5.5 & 4 & \\
\hline Eosinophil levels in serum & 6 & 6 & 7 & \\
\hline IgE levels in serum & 7 & 7 & 7 & \\
\hline Evaluation of exhaled nitric oxide in upper and lower respira & 4 & 9.5 & 3.5 & \\
\hline Mucosal Biopsies / Histopathological specimens & 5 & 6 & 5.5 & \\
\hline Evaluation of nasal lavage & 4 & 6.5 & 4 & 4 \\
\hline Serum Markers & 5 & 6 & 5 & \\
\hline Overall severity of symptoms & 9 & 8.5 & 9 & \\
\hline Disease-specific quality of life & 8 & 8 & 9 & \\
\hline
\end{tabular}

Delphi process.

\section{Outcome definitions}

A patient focus group was convened to determine the patient's perspective on core outcomes, and create definitions for each outcome. Three ENT members of the Steering Group were available to give a clinical overview for each individual outcome on the long-list, but the session was led and supported by an independent, trained facilitator. The facilitator summarised the views of the group and confirmed accuracy prior to concluding the meeting. As the lay definitions were thought to be clear and unambiguous by the Steering group, it was decided that there was no need for additional 'medical definitions'. 
Table 6. Items achieving consensus as essential for inclusion in the core outcome set.

\begin{tabular}{|c|c|c|c|c|}
\hline Item & Little & Moderate & rtance & $\begin{array}{c}\text { Overall \% } \\
\text { Consensus for } \\
\text { Essential Domain }\end{array}$ \\
\hline Compliance with treatment & 0 & 3 & 60 & $95 \%$ \\
\hline Nasal obstruction / blockage/congestion & 0 & 4 & 63 & $94 \%$ \\
\hline Overall control of disease & 0 & 5 & 60 & $92 \%$ \\
\hline Endoscopic appearances & 1 & 4 & 57 & $92 \%$ \\
\hline Acceptability of treatment & 0 & 7 & 56 & $89 \%$ \\
\hline Side effects of treatment (including medical and surgical) & 1 & 8 & 54 & $86 \%$ \\
\hline Overall severity of symptoms & 0 & 10 & 59 & $86 \%$ \\
\hline Disease-specific quality of life & 0 & 9 & 55 & $85 \%$ \\
\hline Frequency of symptoms & 0 & 12 & 56 & $82 \%$ \\
\hline Sense of smell (and taste) & 0 & 12 & 55 & $82 \%$ \\
\hline Length of symptoms & 2 & 13 & 50 & $79 \%$ \\
\hline Need for surgery & 1 & 14 & 50 & $77 \%$ \\
\hline Runny Nose (Nasal discharge) - anterior or posterior & 2 & 15 & 50 & $75 \%$ \\
\hline Ability to perform normal activities & 3 & 13 & 47 & $75 \%$ \\
\hline Length of treatment effect & 0 & 18 & 48 & $72 \%$ \\
\hline
\end{tabular}

Table 7. Items achieving consensus to be excluded from the core outcome set.

\begin{tabular}{|c|c|c|c|c|}
\hline Domain & \multicolumn{3}{|c|}{ No of Stakeholders rating order of Importance } & $\begin{array}{l}\text { Overall \% } \\
\text { Consensus for Un- } \\
\text { important Domain }\end{array}$ \\
\hline Maxillary (cheek) sinus manometry & 45 & 4 & 13 & $73 \%$ \\
\hline
\end{tabular}

\section{Item Prioritization}

We utilised an online Delphi technique (eDelphi software provided by the COMET initiative) inviting patients and healthcare practitioners to individually rate those outcomes from the longlist that are thought to be essential for core outcome sets. There is currently no standard method for sample size calculation in the Delphi process, and thus a pragmatic approach was taken. We had aimed to achieve completed responses in round 2 from at least 100 participants, and therefore, allowing for $10 \%$ attrition between rounds, aimed to recruit at least 110 participants in the first round.

Participants for the Delphi process were recruited from the Steering Group, with further invitations sent to members of the BRS, ERS and ARS, primary care, allergists and respiratory medicine through the BSACl. Patient participants were recruited from clinical practice. Ethical approval for patient participation in the Delphi process and focus groups was granted.

In the first round, each participant was asked to consider each outcome item, and to decide which were essential to the core outcome set. By using the 10 point scale proposed by the GRADE group (17), where 1 to 3 signifies an outcome of limited importance, 4 to 6 important but not critical, and 7 to 9 critical
(10), participants were able to rate the importance of each item and hence inclusion within the core outcome set. Participants were further asked to submit any outcomes thought to be important but missing from the long-list.

Additional outcomes listed by participants were reviewed and coded by two members of the study team ( $\mathrm{RH}$ and $\mathrm{CH}$ ) and new outcomes were included in round 2 . In the second round, participants were presented with the aggregate results of ratings for each item (the median score for each item was displayed, separately for each stakeholder group and for the combined cohort), allowing an opportunity of reflection, and were asked to consider each outcome again, using the same scoring system ranking each on a nine-point Likert scale.

Consensus regarding whether an outcome should be included in the $\operatorname{COS}$ was defined a priori as $70 \%$ or more of the respondents scoring it 7 to 9 and fewer than $15 \%$ scoring it as 1 to 3 . Consensus that an outcome should not be included in the COS was defined as $70 \%$ or more scoring it as 1 to 3 and fewer than $15 \%$ scoring it as 7 to 9 . All other score distributions would be taken to indicate lack of agreement for inclusion of a given outcome in the COS. In the event that different stakeholder groups 
Table 8. Items failing to reach $70 \%$ consensus, but rated by the majority to be essential - may be considered in extended sets.

\begin{tabular}{|c|c|c|c|c|}
\hline \multirow[t]{2}{*}{ Item } & \multicolumn{3}{|c|}{ No of Stakeholders rating order of Importance } & \multirow{2}{*}{$\begin{array}{c}\text { Overall \% } \\
\text { Consensus for } \\
\text { Essential Domain }\end{array}$} \\
\hline & Little & Moderate & Very & \\
\hline Ongoing treatment requirements / Healthcare utilisation & 2 & 19 & 44 & $68 \%$ \\
\hline Radiological evaluation & 3 & 18 & 41 & $66 \%$ \\
\hline Complete resolution of symptoms & 1 & 23 & 42 & $65 \%$ \\
\hline Global quality of life & 2 & 21 & 42 & $65 \%$ \\
\hline Lower respiratory (chest) symptoms & 0 & 24 & 38 & $61 \%$ \\
\hline Treatment preference & 0 & 25 & 38 & $60 \%$ \\
\hline Facial pain / pressure & 3 & 24 & 40 & $59 \%$ \\
\hline IgE levels in serum & 3 & 27 & 32 & $52 \%$ \\
\hline
\end{tabular}

Table 9. Remaining items - Items did not reach consensus definition for inclusion in the core outcome set, and were rated by majority of respondents as moderately important.

\begin{tabular}{|c|c|c|c|c|}
\hline \multirow[t]{2}{*}{ Item } & \multicolumn{3}{|c|}{ No of Stakeholders rating order of Importance } & \multirow{2}{*}{$\begin{array}{l}\text { Overall \% } \\
\text { Consensus for } \\
\text { Moderate Domain }\end{array}$} \\
\hline & Little & Moderate & Very & \\
\hline Itching /Sneezing & 5 & 48 & 16 & $81 \%$ \\
\hline Costs of treatment to third party payers & 4 & 42 & 17 & $67 \%$ \\
\hline Mucosal Biopsies / Histopathological specimens & 6 & 40 & 16 & $65 \%$ \\
\hline Headache & 8 & 41 & 16 & $63 \%$ \\
\hline (Nose Bleeds) Epistaxis & 21 & 41 & 6 & $60 \%$ \\
\hline Cough & 13 & 39 & 13 & $60 \%$ \\
\hline Mental ill-health & 12 & 37 & 14 & $59 \%$ \\
\hline Costs of treatment to patients & 1 & 37 & 25 & $59 \%$ \\
\hline Eye symptoms eg. dryness; itching; irritation & 18 & 38 & 10 & $58 \%$ \\
\hline Clinical measures of lower respiratory (chest) function & 4 & 36 & 22 & $58 \%$ \\
\hline Evaluation of sense of smell & 8 & 36 & 18 & $58 \%$ \\
\hline Change in the individual microbiome & 7 & 36 & 19 & $58 \%$ \\
\hline Emotional wellbeing and stability & 8 & 36 & 19 & $57 \%$ \\
\hline Serum Markers & 13 & 35 & 14 & $56 \%$ \\
\hline Dryness (nose or mouth) & 22 & 36 & 7 & $55 \%$ \\
\hline Ear symptoms; eg. dizziness; fullness & 22 & 37 & 9 & $54 \%$ \\
\hline Medication usage for lower respiratory (chest) disease & 1 & 33 & 28 & $53 \%$ \\
\hline Hospitalisation for chest problems & 4 & 33 & 25 & $53 \%$ \\
\hline Evaluation of the nasal airway using special tests & 15 & 33 & 14 & $53 \%$ \\
\hline Fatigue & 13 & 35 & 20 & $51 \%$ \\
\hline Bad breath and taste in the mouth & 25 & 33 & 7 & $51 \%$ \\
\hline Evaluation of mucociliary function & 16 & 31 & 15 & $50 \%$ \\
\hline Eosinophil levels in serum & 2 & 31 & 29 & $50 \%$ \\
\hline Resistance to antibiotics in the community & 5 & 30 & 27 & $48 \%$ \\
\hline Histological evidence of fungal hyphae & 11 & 29 & 22 & $47 \%$ \\
\hline Sleep disturbance & 8 & 30 & 28 & $45 \%$ \\
\hline Evaluation of nasal lavage & 23 & 24 & 15 & $39 \%$ \\
\hline Exhaled Nitric Oxide in upper \& lower respiratory tract & 24 & 23 & 15 & $38 \%$ \\
\hline
\end{tabular}


Table 10. Final Item list for inclusion in Core Outcome Set for CRS, including proposed measurement tools

\begin{tabular}{|c|c|c|}
\hline Domain & Item & Proposed measurement tool \\
\hline $\begin{array}{l}\text { Patient reported } \\
\text { symptoms and QOL }\end{array}$ & $\begin{array}{l}\text { Overall symptom severity } \\
\text { Frequency of symptoms } \\
\text { Duration of symptoms } \\
\text { Duration of treatment effect } \\
\text { Sense of smell } \\
\text { Runny nose / Nasal discharge (anterior or } \\
\text { posterior) } \\
\text { Nasal obstruction / blockage/congestion } \\
\text { Disease specific quality of life }\end{array}$ & $\begin{array}{l}\text { SNOT-22 repeated over time } \\
\text { Additional question required to address fre- } \\
\text { quency of symptoms }\end{array}$ \\
\hline \multirow[t]{3}{*}{ Control of disease } & Overall control of disease & $\begin{array}{l}\text { Need for systemic medication (steroid or } \\
\text { antibiotic) }\end{array}$ \\
\hline & Need for surgery & Progression to surgery \\
\hline & $\begin{array}{l}\text { Endoscopic appearances (including presence/ } \\
\text { quality of pus, presence and size of polyps, } \\
\text { oedema, crusting, inflammation) }\end{array}$ & Lund-Kennedy score \\
\hline Impact on daily activity & Ability to perform normal activities & $\begin{array}{l}\text { SNOT-22 } \\
\text { (or specific measures of productivity) }\end{array}$ \\
\hline Acceptability of treatment and side effects & $\begin{array}{l}\text { Compliance with treatment } \\
\text { Acceptability of treatment } \\
\text { Side effects of treatment (including medical } \\
\text { and surgical) }\end{array}$ & Measurement of compliance and side effects \\
\hline
\end{tabular}

disagreed about the inclusion of specific outcomes in the COS, only items about which there was consensus were included.

Two rounds of the Delphi process were initially planned, with stability measured by consistency of responses between successive rounds of a study and used to determine the value of further rounds ${ }^{(18)}$. The Steering group and two patient representatives considered the outcomes of each round of the Delphi, and approved the decision to end the Delphi process and the final core outcome set.

\section{Results}

\section{Longlist development}

The SR identified a total of 83 randomised control trial papers and 8 Cochrane reviews. Of these, 14 trials were excluded, as they were trials that included either paediatric patients, those with allergic rhinitis or published conference abstracts. A total of 69 RCTs were finally obtained and included within this study. No further references were identified through examination of the bibliography of the published systematic reviews or trials. Three hundred and sixty five individual outcomes were extracted from the clinical trials. The trials overlapped in their use of outcomes and between them had used 68 different outcomes and outcome measures. These were then mapped onto twenty-three pre-determined sub-categories, belonging to nine main core categories (Table 1).

The OMIPP project generated 653 suggestions of important outcomes from 235 participants. Of these 549 ( 169 from people with rhinosinusitis, and 380 from practitioners) fitted our description of an outcome. These were mapped onto eight domains and combined with the longlist above (Figure 1; responses grouped under main domains). Of note there was consistency with the domains identified in the SR and those identified with qualitative analysis of the response to the OMIPP project

Finally, a focus group of 10 patients with CRS and the Steering group reviewed the list to ensure that no missing items could be identified. A final long-list of 54 items, mapped onto 9 domains was developed (Table 2).

\section{Delphi Process}

The first round of the Delphi process was held in April 2017. One hundred and fourteen participants were recruited, achieving our initial target; this included 8 Steering Group members, 19 patients, and 88 physicians from primary care/allergy/ENT, with ENT surgeons and allergy accounting for $91 \%$ of the latter group. There was close agreement between the Steering group and other healthcare providers (HCPs) (Table 3). In contrast, there were marked differences in responses amongst patients; while patients also rated those items thought by both the Steering Group and other HCPs to be essential to a COS, they also rated almost all clinical measures as essential, even though these were not rated highly by clinicians. Fifteen items were considered essential to the core outcome set by all respondents.

Unfortunately, there was a significant drop-out in respondents between round 1 and 2, despite sending multiple reminders. At round 2, we received 67 replies, representing $59 \%$ of our initial 
cohort. There was a high level of stability in the overall median scores for each item from round 1 to round 2 (Table 4). The overall category (not important / moderate importance / essential to COS) only changed in 4 out of 54 items. Twenty-three items had a median score rating the item as essential to the core outcome set. There was much greater consistency in categorical rating between stakeholder groups and it was agreed that there was no need for a third round (Table 5).

When looking at items that met our definition of consensus, 15 items were considered essential to the core outcome set (Table 6), with $>70 \%$ rating as 7 or more, and less than $15 \%$ rating as 3 or less; 1 item was agreed to be unimportant (Table 7). Overall, 8 items were rated as essential by the majority of respondents but did not reach the $70 \%$ threshold (Table 8), while the remainder were considered to be of moderate importance only, by the majority (Table 9).

Thus the final core-outcome set contains 15 items, over 4 domains (Table 10).

\section{Discussion}

While at first glance this list may seem to be too extensive, the items are spread over 4 key domains. Three key patient rated symptoms: nasal obstruction, nasal discharge and sense of smell are included, alongside overall symptom severity and both frequency and duration of symptoms. Repeated use over time of a PROM that includes these symptoms will allow both the symptom scores and duration of symptoms as well as duration of treatment effect to be measured. PROMs such as the SNOT-22 also include items on ability to perform normal daily activity and will evaluate overall disease specific quality of life.

A measure of treatment compliance, alongside evaluation of the side effects of any treatment should be included in future trials, and likely obviates the need for a separate question on acceptability of treatment. Endoscopy scores were considered essential within the outcome set. This is most commonly graded using the Lund-Kennedy score.

Finally a measure of disease control was thought to be important. Control of disease is a concept that combines current disease status or symptom burden and the level of intervention required to get there. The Sinus Control Test is made up of 4 questions, including severity of nasal discharge, nasal obstruction, frequency of symptoms that interfere with normal activity and need for systemic medication in the last 2 weeks ${ }^{(19)}$. The EPOS guidelines ${ }^{(16)}$ defined uncontrolled disease as the presence of 3 or more of the following; 'bothersome' symptoms of nasal obstruction, discharge, facial pain anosmia, sleep disturbance, abnormal endoscopy or systemic medication needed to control disease. This definition was evaluated by Harvey et al., who found better agreement with physician and patient rated control using a modified version of only 3 items; severity of nasal obstruction, abnormal endoscopy, and requirement for systemic medication in the last 3 months (the NOSE staging system of disease control) ${ }^{(20)}$. Key to disease control in all of these control measures is the need for systemic medications - either oral steroids or antibiotics, which could be evaluated alongside the SNOT-22. The need for surgical intervention was rated as an essential outcome in the $\mathrm{COS}$ and is also a reflection for disease control. Indeed, a number of trials for biological agents are including avoidance of surgery as an outcome, and particularly in terms of cost-effectiveness evaluation this will be a great importance. Currently surgery represents the highest costs in the patient pathway with CRS, although this may change in the era of monoclonal antibodies.

Many aspects of the COS are captured by the SNOT-22; a modification of the SNOT-22 to include a question of need for systemic medication or progression to surgery, compliance with and side effects of treatment, more information on frequency of symptoms and impact on ability to perform normal activities would better meet the needs of the COS. Of course, any modification of the SNOT-22 from its current form would require further re-validation. Thus, although 15 individual items were agreed to be important in the COS, these could be measured by a single PROM repeated over time, in conjunction with the Lund-Kennedy endoscopic score.

This project aimed to define what outcomes should be included in a COS. The Steering Group agreed that it was important to make provisional recommendations regarding how these outcomes should be measured. Further work is now required to further develop and revalidate existing outcome measures.

One strength of this project is the extensive work to develop the long-list of outcomes, including thematic analysis of views of patients and practitioners and a systematic review of outcomes used in CRS research. Our major limitation is that the response rate between Delphi rounds was lower than expected. This was in part due to delays in between rounds and problems using the electronic Delphi tool, which was newly launched at the time of use. We had planned to undertake 3 rounds, but analysis suggested that stability had been reached and that further rounds, with risk of greater drop out would be unlikely to significantly change the final COS.

It is not intended that these core outcomes are the only outcomes that will be measured by trials of interventions for CRS, and researchers can of course add additional outcomes to suit the needs of specific trials. However, we hope that inclusion of these 
core outcomes in all future trials will increase the value of future research on interventions for CRS in adults.

\section{Implementation}

We plan to seek endorsement from the European and American Rhinologic Societies in the first instance and ask for the COS to be included in future iterations of International guidelines such as EPOS and ICARS ${ }^{(21)}$. We will make contact with major funding bodies (eg. NIHR in the UK and NIH in North America) to encourage inclusion of core outcomes in trials at the point of funding.

\section{Authorship contribution}

$\mathrm{CH}$ design of project, data collection, manuscript preparation; $\mathrm{RH}, \mathrm{ASJ}, \mathrm{RL}$ - data collection; All other authors - Steering committee; expert opinion, oversight of project, review and approval of final manuscript.

\section{Conflict of interest}

No conflicts of interest to disclose.

\section{References}

1. Hastan D, Fokkens WJ, Bachert C, Newson RB, Bislimovska J, Bockelbrink $A$, et al. Chronic rhinosinusitis in Europe - an underestimated disease. A GA(2) LEN study. Allergy. 2011;66(9):1216-23.

2. Gliklich RE, Metson R. The health impact of chronic sinusitis in patients seeking otolaryngologic care. Otolaryngol Head Neck Surg. 1995:113(1):104-9.

3. Gulliford MC, Dregan A, Moore MV Ashworth M, Staa T, McCann G, et al. Continued high rates of antibiotic prescribing to adults with respiratory tract infection: survey of 568 UK general practices. BM Open. 2014;4(10):e006245.

4. Caulley L, Thavorn K, Rudmik L, Cameron C, Kilty SJ. Direct costs of adult chronic rhinosinusitis by using 4 methods of estimation: Results of the US Medical Expenditure Panel Survey. J Allergy Clin Immunol. 2015;136(6):1517-22.

5. Smith KV, Orlandi RR, Rudmik L. Cost of adult chronic rhinosinusitis: A Systematic review. Laryngoscopy. 2015;125(7):1547-56.

6. Rudmik L, Bird C, Dean S, Dort JC, Schorn R, Kukec E. Geographic Variation of Endoscopic Sinus Surgery in Canada: An Alberta-Based Small Area Variation Analysis. Otolaryngol Head Neck Surg. 2015;153(5):865-74

7. Rudmik L, Holy CE, Smith TL. Geographic variation of endoscopic sinus surgery in the United States. Laryngoscope. 2015:125(8):1772-8.

8. (WHO) WHO. WHO: WHO handbook for guideline development 2010 [Available from: http://wwwwhoint/hiv/topics/mtct/ grc_handbook_mar2010_1pdf.

9. Rosenbaum SE, Glenton C, Nylund HK,
Oxman AD. Summary-of-findings tables in key informaiton. J Clin Epidemiol. 2010;63:620-6.

10. Williamson PR, Altman DG, Blazeby JM, Clarke M, Devane D, Gargon E, et al. Developing core outcome sets for clinical trials: issues to consider. Trials. 2012;13:132.

11. Kirkham JJ, Davis K, Altman DG, Blazeby JM, Clarke M, Tunis S, et al. Core Outcome Set-STAndards for Development: The COS-STAD recommendations. PLOS Med. 2017;14(11):e1002447.

12. Kirkham JJ, Gorst S, Altman DG, Blazeby JM, Clarke M, Devane D, et al. Core Outcome Set-STAndards for Reporting: The COS-STAR Statement. PLOS Med. 2016;13(10):e1002148.

13. Soni-Jaiswal A, Lakhani R, Hopkins C. Developing a core outcome set for chronic rhinosinusitis: a systematic review of outcomes utilised in the current literature. Trials. 2017;18(1):320.

14. Hopkins C, Philpott C, Crowe S, Regan S, Degun A, Papachristou I, et al. Identifying the most important outcomes for systematic reviews of interventions for rhinosinusitis in adults: working with Patients, Public and Practitioners. Rhinology. 2016;54(1):20-6.

15. Rudmik L, Hopkins C, Peters A, Smith TL, Schlosser RJ, Soler ZM. Patient-reported outcome measures for adult chronic rhinosinusitis: A systematic review and quality assessment. J Allergy Clin Immunol. 2015;136(6):1532-40 e1-2.

16. Fokkens WJ, Lund VJ, Mullol J, Bachert C Alobid I, Baroody F, et al. EPOS 2012: European position paper on rhinosinusitis and nasal polyps 2012. A summary for otorhinolaryngologists. Rhinology. 2012;50(1):1-12
17. The GRADE working group 2004-2017 [Available from: http://www.gradeworkinggroup.org.

18. Knott A, Pathak S, McGrath JS, Kennedy R, Horgan A, Mythen $M$, et al. Consensus views on implementation and measurement of enhanced recovery after surgery in England: Delphi study. BMJ Open. 2012;2(6).

19. Banglawala SM, Schlosser RJ, Morella K, Chandra R, Khetani J, Poetker DM, et al. Qualitative development of the sinus control test: a survey evaluating sinus symptom control. Int Forum Allergy Rhinol. 2016;6(5):491-9.

20. Snidvongs K, Heller GZ, Sacks R, Harvey RJ. Validity of European position paper on rhinosinusitis disease control assessment and modifications in chronic rhinosinusitis. Otolaryngol Head Neck Surg. 2014;150(3):479-86.

21. Orlandi RR, Kingdom TT, Hwang PH, Smith TL, Alt JA, Baroody FM, et al. International Consensus Statement on Allergy and Rhinology: Rhinosinusitis. Int Forum Allergy Rhinol. 2016;6 Suppl 1:S22-209.

\section{Claire Hopkins}

Professor of Rhinology

ENT Department

Guy's Hospital

SE1 9RT London

United Kingdom

E-mail: claire.hopkins@gstt.nhs.uk 